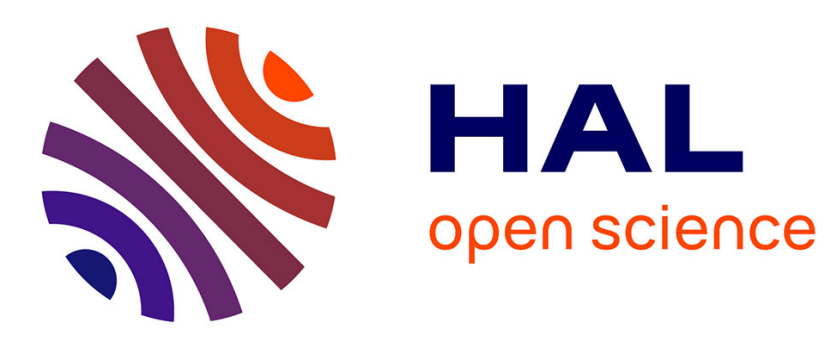

\title{
Robust control/scheduling co-design: application to robot control
}

Daniel Simon, David Robert, Olivier Sename

\section{To cite this version:}

Daniel Simon, David Robert, Olivier Sename. Robust control/scheduling co-design: application to robot control. RTAS'05 11th IEEE Real-Time and Embedded Technology and Applications Symposium, IEEE, Mar 2005, San Francisco, CA, USA. inria-00000733

\section{HAL Id: inria-00000733 \\ https://hal.inria.fr/inria-00000733}

Submitted on 15 Nov 2005

HAL is a multi-disciplinary open access archive for the deposit and dissemination of scientific research documents, whether they are published or not. The documents may come from teaching and research institutions in France or abroad, or from public or private research centers.
L'archive ouverte pluridisciplinaire HAL, est destinée au dépôt et à la diffusion de documents scientifiques de niveau recherche, publiés ou non, émanant des établissements d'enseignement et de recherche français ou étrangers, des laboratoires publics ou privés. 


\section{Robust control/scheduling co-design: application to robot control}

\author{
Daniel Simon \\ INRIA Rhône-Alpes \\ 655 avenue de l'Europe, Montbonnot \\ 38334 Saint-Ismier Cedex, France \\ Daniel.Simon@inrialpes.fr
}

\author{
David Robert, Olivier Sename \\ Laboratoire d'Automatique de Grenoble \\ ENSIEG-BP 46 \\ 38402 Saint Martin d'Hères Cedex, France \\ \{david.robert, olivier.sename\}@inpg.fr
}

\begin{abstract}
Control systems running on a computer are subject to timing disturbances coming from implementation constraints. Fortunately closed-loop systems behave robustly w.r.t. modelling errors and disturbances, and the controller design can be performed to explicitly enhance robustness against specific uncertainties. On one hand robustness in process controllers can be used to comply with weakly modelled timing uncertainties. On the other hand the principle of robust closed-loop control can also be applied to the real-time scheduler to provide on-line adaption of some scheduling parameters, with the objective of controlling the computing resource allocation. The control performance specification may be set according to both control and implementation constraints. The approach is illustrated through several examples using simulation and an experimental feedback scheduler is briefly described.
\end{abstract}

Keywords: control/scheduling co-design, feed-back scheduling, robustness, resource management

\section{Introduction}

Digital control systems can be implemented as a set of tasks running on top of an off-the-shelf real-time operating system (RTOS) using fixed-priority and preemption. The performance of the control, e.g measured by the tracking error, and even more importantly its stability, strongly relies on the values of the sampling rates and sensor-to-actuator latencies (the latency we consider for control purpose is the delay between the instant when a measure $q_{n}$ is taken on a sensor and the instant when the control signal $U\left(q_{n}\right)$ is received by the actuators [1]). Therefore it is essential that the implementation of the controller respect an adequate temporal behaviour to meet the expected performance. However implementation constraints such as multi-rate sampling, preemption, synchronisation and various sources of delays makes the run-time behaviour of the controller very difficult to accurately predict. However as we deal with closed-loop controllers we may take advantage of the robustness of such systems to design and implement flexible and adaptive real-time control architectures.

This paper deals with robust and adaptive solutions for real-time scheduling and control co-design. In the next section we review some properties of closed-loop controllers in contrast with real-time implementation constraints. Some recent results in control and scheduling co-design are recalled in section 3 . Section 4 gives an overview of a new feedback scheduling strategy aimed to on-line adapt the tasks period according to the computing resource activity. This approach is then applied in the design of a robot controller in section 5, for which the importance of integrated control/scheduling co-design is emphasised. Finally an experimental feedback scheduler implementation is described in section 6 and future research directions conclude the paper.

\section{Problem position: background on control and implementation con- straints}

Closed-loop digital control systems use a computer to periodically sample sensors, compute a control law and send control signals to the actuators of a continuous time physical process. The control algorithm can be either designed in continuous time and then discretized or directly synthesised in discrete time taking account of a model of the plant sampled by a zeroorder holder. Control theory for linear systems sampled at fixed rates has been established a long time ago, e.g. [1].

Assigning an adequate value for the sampling rate is a decisive duty as this value has a direct impact on the control performance and stability. While an abso- 
lute lower limit for the sampling rate is given by Shannon's theorem, in practise rules of thumb are used to give a useful range of control frequencies according to the process dynamics and to the desired closed-loop bandwidth (see for example section 5.3). A general rule is that decreasing the control period and latencies allows for improved control performance, e.g. measured by the tracking error or disturbances rejection.

\subsection{Digital control of continuous systems}

To implement a controller the basic idea consist in running the whole set of control equations in a unique periodic real-time task which clock gives the controller sampling rate. In fact all parts of the control algorithm do not have an equal weight and urgency w.r.t. the control performance. To minimise the latency a control law can be basically implemented as two real-time blocks, the urgent one sends the control signal directly computed from the sampled measures while updating the state estimation or parameters can be delayed or even more computed less frequently [1].

In fact, a complex system involves sub-systems with different dynamics which must be further coordinated [18]. Assigning different periods and priorities to different blocks according to their relative weight allows for a better control of critical latencies and for a more efficient use of the computing resource [15]. However in such cases finding adequate periods for each block is out of the scope of current control theory and must be done through case studies, simulation and experiments.

\subsection{Control and timing uncertainty}

While timing uncertainties have an impact on the control performance they are difficult to be accurately modelled or constrained to lie inside precisely known bounds. Thus it is worth examining the sensitivity of control systems w.r.t. timing fluctuations.

Control systems are often cited as examples of "hard real-time systems" where jitter and deadline violations are strictly forbidden. In fact experiments show that this assumption may be false for closed-loop control. Any practical feedback system is designed to obtain some stability margin and robustness w.r.t. the plant parameters uncertainty. This also provides robustness w.r.t. timing uncertainties: closed-loop systems are able to tolerate some amount of sampling period and computing delays deviations, jitter and occasional data loss with no loss of stability or integrity, e.g. [4]: their behaviour can still be considered as correct as long as the sample-induced disturbances stay inside the performance specification bounds.

Therefore the hard real-time assumption can softened to better cope with the reality of closed-loop control. For example they can be changed for "weakly hard" constraints: absolute deadlines are replaced by statistical ones, e.g. the allowable output jitter compliant with the desired control performance or the number of allowed deadlines miss over a specified time window [2]. Note that to be fully exploited weakly hard constraints should be associated with a decisional process: tasks missing their deadline can be for example delayed, aborted or skipped according to their impact on the control law behaviour.

Finding the values of such weakly hard constraints for a given control law is currently out of the scope of current control theory in the general case. However the intrinsic robustness of closed-loop controllers allows for complying with softened timing constraints specification and flexible scheduling design.

\subsection{Control and scheduling}

Usually, real-time systems are modelled by a set of recurrent tasks assigned to one or several processors and a worst case response times technique is used to analyse fixed-priority real-time systems. Well known scheduling policies, such as Rate Monotonic for fixed priorities and EDF for dynamic priorities, assign priorities according to timing parameters, respectively sampling periods and deadlines. They are said to be "optimal" as they maximise the number of tasks sets which can be scheduled with respect of deadlines, under some restrictive assumptions. Unfortunately they are not optimised for control purpose.

They hardly take into account precedence and synchronisation constraints which naturally appear in a control algorithm. The relative urgency or criticality of the control tasks can be unrelated with the timing parameters. Thus, the timing requirements of control systems w.r.t. the performance specification do not fit well with scheduling policies purely based on schedulability tests. It has been shown through experiments, e.g. [4], that a blind use of such traditional scheduling policy can lead to an inefficient controller implementation; on the other hand a scheduling policy based on application's requirements, associated with a right partition of the control algorithm into real-time modules may give better results.

Another example of unsuitability between computing and control requirements arises when using priority inheritance or priority ceiling protocols to bypass priority inversion due to mutual exclusion, e.g. to en- 
sure the integrity of shared data. While they are designed to avoid dead-locks and minimise priority inversion lengths, such protocols jeopardise at run-time the initial schedule which was carefully designed to meet control requirements. As a consequence latencies along some control paths can be largely increased leading to a poor control performance or even instability.

Finally off-line schedulability analysis rely on a right estimation of the tasks worst case execution time. Even in embedded systems the processors use caches and pipelines to improve the average computing speed while decreasing the timing predictability. Another source of uncertainty may come from some pieces of the control algorithm. For example, the duration of a vision process highly depends on incoming data from a dynamic scene. Also some algorithms are iterative with a badly known convergence rate, so that the time before reaching a predefined threshold is unknown (and must be bounded by a timeout). In a dynamic environment some control activities can be suspended or resumed and control algorithms with different costs can be scheduled according to various control modes leading to large variations in the computing load.

Thus real-time control design based on worst case execution time, maximum expected delay and strict deadlines inevitably leads to a low average usage of the computing resource.

\section{Related work}

Control/scheduling co-design This mainly concerns the integration of control performance knowledge in the scheduling parameters assignment. Indeed, once a control algorithm has been designed, a first job consists in assigning timing parameters, i.e. periods of tasks and deadlines, so that the controller's implementation satisfies the control objective. This may be done off-line or on-line.

In off-line control/scheduling co-design setting adequate values for the timing parameters rapidly falls into case studies based on simulation and experiments. For instance in [11] off-line iterative optimisation is used to compute an adequate setting of periods, latencies and gains resulting in a requested control performance according to the available computing resource and implementation constraints. Also in [12] the temporal requirements of the control system are described using complex temporal attributes (e.g. nominal period and allowed variations, precedence constraints... ): this model is then used by an off-line iterative heuristic procedure to assign the scheduling parameters (e.g. priorities and offsets) to meet the constraints.
Concerning co-design for on-line implementation, recent results deal with varying sampling rates in control loops in the framework of linear systems: for example [13] show that, while switching between two stable controllers, too frequent control period switches may lead to unstability. Unfortunately most real-life systems are non-linear and the extrapolation of timing assignment through linearising often gives rough estimations of allowable periods and latencies or even can be meaningless. In fact, as it will be shown in the robot control application, the plant knowledge is necessary to get an efficient control/scheduling co-design.

Feedback scheduling Besides traditional assignment of fixed scheduling parameters more flexible scheduling policies have been investigated. Let us cite e.g. [3] where the elasticity of the tasks' periods enables for controlling the quality of service of the system as a function of the current estimated load. While such an approach is still working in open loop w.r.t. a controlled plant, the on-line combination the control performance and implementation constraints lead to the feedback scheduling approach.

This new approach has been initiated both from the real-time computing side [10] and from the control side $[5,7,6]$. The idea consists in adding to the process controller an outer sampled feedback loop ("scheduling regulator") to control the scheduling parameters as a function of a QoC (Quality of Control) measure. It is expected that an on line adaption of the scheduling parameters of the controller may increase its overall efficiency w.r.t. timing uncertainties coming from the unknown controlled environment. Also we know from control theory that closing the loop may increase performance and robustness against disturbances when properly designed and tuned (otherwise it may lead to instability).

Figure 1 gives an general overview of a feed-back scheduler where an outer loop (the scheduling controller) adapts in real-time the scheduling parameters from measurements taken on the computer's activity, e.g. the computing load . Besides this controller working periodically (at a rate larger than the sampling periods of the plant control tasks), the system's structure may evolve along a discrete time scale upon occurrence of events, e.g. for new tasks admission or exception handling. These decisional processes may be handled by another real-time task, the scheduling manager, which is not further detailed in this paper. Notice that such a manager may give a reference to the controller resource utilisation.

The design problem can thus be stated as control performance optimisation under constraint of available computing resources. Major studies result from $[8,4]$ 


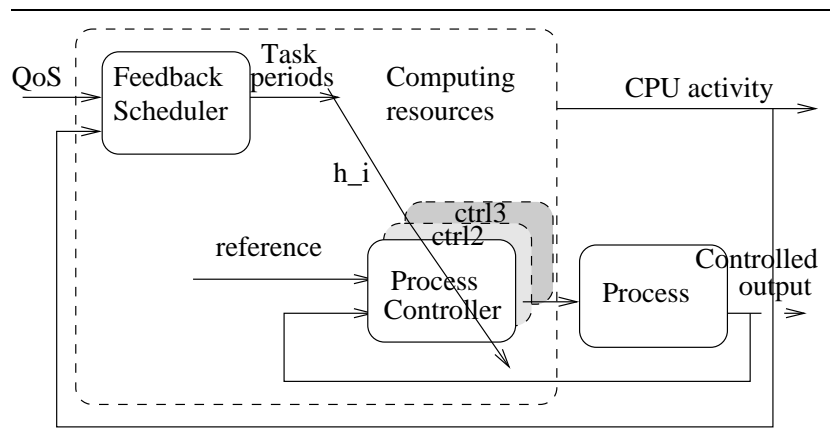

Figure 1. Hierarchical control structure

where it is suggested that a simple solution to this optimal control problem (i.e. under resource constraint) is the calculation of the new task periods by the rescaling:

$$
h_{i}^{\text {new }}=h_{i_{\text {nominal }}} \frac{U}{U_{s p}}
$$

where $U_{s p}$ is the utilisation set-point. The feedback scheduler then controls the processor utilisation by assigning task periods that optimise the overall control performance.

Preliminary works have been done by the authors. In [14] an LQG approach is used to design the feedback scheduling while in [17], an $H_{\infty}$ control problem is solved for a two tasks systems (without differentiation between both tasks). In what follows the proposed methodology is described.

\section{A new methodology for Feed-back scheduling}

Feedback scheduling is a dynamic approach allowing to better use the computing resources, in particular when the workload changes e.g. due to the activation of an admitted new task. Indeed, the CPU activity will be controlled according to the resource availability by adjusting scheduling parameters (i.e. period) of the plant control tasks.

In the approach here proposed, a way to take into account the resource sharing over a multitasks process is developed. In what follows, the control design issue is described including the control structure, the specification of control inputs and measured outputs, as well as the modelling step.

\subsection{Control structure}

In Fig 2 scheduling is viewed as a dynamical system between control task frequencies and processor utilisation. As far as the adaptation of the control tasks is concerned, the load of the other tasks is seen as an output disturbance.

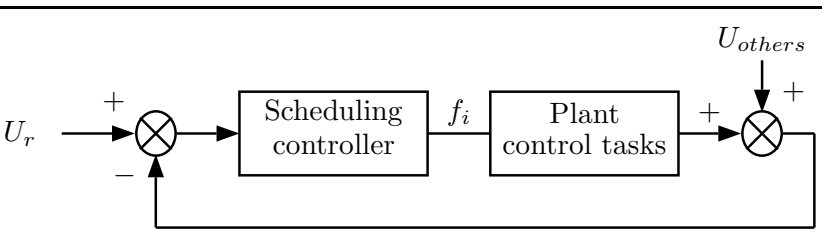

Figure 2. Feedback scheduling bloc diagram

\subsection{Sensors and actuators}

As stated in section 2.3, priorities must be assigned to control tasks according to their relative urgency ; this ordering remains the same in the case of a dynamic scheduler. Dynamic priorities, e.g. as used in EDF, only alter the interleaving of running tasks and will fail in adjusting the computing load w.r.t. the control requirements. In consequence we have elected the tasks periods to be the main actuators of the system running on top of a fixed priority scheduler ${ }^{1}$.

As the aim is to adjust on-line the sampling periods of the controllers in order to meet the computing resource requirements, the control inputs are thus the periods of the control tasks.

The measured output is the CPU utilisation. Let us first recall that the scheduling is here limited to periodic tasks. In this case the processor load induced by a task is defined by $U=\frac{c}{h}$ where $c$ and $h$ are the execution time and period of the task. Hence processor load induced by a task is estimated, in a similar to way [6], for each period $h_{s}$ of the scheduling controller, as :

$$
\hat{U}_{k h_{s}}=\lambda \hat{U}_{(k-1) h_{s}}+(1-\lambda) \frac{\bar{c}_{k h_{s}}}{h_{(k-1) h_{s}}}
$$

where $h$ is the sampling frequency currently assigned to the plant control task (i.e. at each sampling instant $k h_{s}$ ) and $\bar{c}$ is the mean of its measured job executiontime. $\lambda$ is a forgetting factor used to smooth the measure.

\subsection{Control design and implementation}

The proposed control design method for feedback scheduling is here developed. First one should note

1 Possible secondary actuators are variants of the control algorithms, with different QoS contributions to the whole system. Such variants should be handled by the scheduling manager working on a discrete events time scale 
that, as shown in [17], if the execution times are constant, then the relation, $U=\sum_{i=1}^{n} C_{i} f_{i}$ (where $f_{i}=$ $1 / h_{i}$ is the frequency of the task) is a linear function (while it would not be as a function of the task periods). Therefore, using (1), the estimated CPU load is given as:

$$
\hat{U}\left(k h_{S}\right)=\frac{(1-\lambda)}{z-\lambda} \sum_{i=1}^{n} \bar{c}_{i}\left(k h_{S}\right) f_{i}\left(k h_{S}\right)
$$

As $\bar{c}$ depends on the runtime environment (e.g. processor speed) a "normalised" linear model of the task $i$ (i.e independent on the execution time),$G_{i}$, is used for the scheduling controller synthesis where $\bar{c}$ is omitted and will be compensated by on-line gain-scheduling $(1 / \bar{c})$ as shown below.

$$
G_{i}(z)=\frac{\hat{U}(z)}{f_{i}(z)}=\frac{1-\lambda}{z-\lambda}, \quad 1=1, \ldots, n
$$

As illustration, in a single control task system, the control scheme is therefore as in figure 3 where the estimated execution-times are used on-line to adapt the gain of the controller for the original CPU system (2) (this allows to compensate the variations of the job execution time).

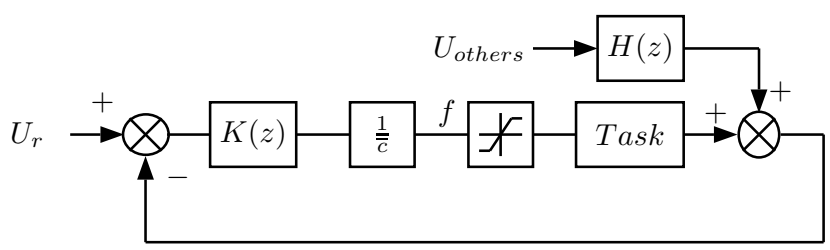

Figure 3. Control scheme for CPU resources

According to this control scheme, the design of the controller $K$ can be made using any advanced control methodology. For the considered application (see section 5), we have chosen the well known $H_{\infty}$ control theory which can lead to a robust controller w.r.t modelling errors (see [19] for details on $H_{\infty}$ control). Moreover it provides good properties in the presence of external disturbance, as it is emphasised in the illustrative examples.

\section{Integrated control-scheduling co- design in robot control}

We consider here a seven degrees of freedom Mitsubishi PA10 robot arm that has been previously modelled and calibrated [16].

\subsection{Plant modelling and control structure}

The problem under consideration is to track a desired trajectory for the position of the end-effector. Using the Lagrange formalism the following model can be obtained:

$$
\Gamma=M(q) \ddot{q}+\operatorname{Gra}(q)+C(q, \dot{q})
$$

where $q$ stands for the positions of the joints, $M$ is the inertia matrix, Gra is the gravity forces vector and $C$ gathers Coriolis, centrifugal and friction forces.

The structure of the (ideal) linearising controller includes a compensation of the gravity, Coriolis/centrifugal effect and Inertia variations as well as a Proportional-Derivative (PD) controller for the tracking and stabilisation problem, of the form:

$$
\Gamma=\operatorname{Gra}(q)+C(q, \dot{q})+K_{p}\left(q_{d}-q\right)+K_{d}\left(\dot{q}_{d}-\dot{q}\right),
$$

leading to the linear closed-loop system $M(q) \ddot{q}=$ $K_{p}\left(q_{d}-q\right)+K_{d}\left(\dot{q}_{d}-\dot{q}\right)$.

This controller is divided in four tasks, i.e. a specific task is considered for the PD control, for the gravity, Inertia and Coriolis compensations, in order to use a multi-rate controller. In this first cautious feedback scheduling scheme, only the periods of the compensation tasks will be adapted, as they have a moderate impact on the closed-loop stability while they are more time consuming compared with the PD task.

\subsection{A first step to co-design: evaluation of controller cost functions}

In the co-design, the aim is to give the precedence (i.e. more resources) to the tasks that are more important for the robot control. To evaluate this importance a cost function is defined as

$$
J=\int_{0}^{t_{\text {trajectory }}} \sum_{i=1}^{i=7}\left(q_{i}-q d_{i}\right)^{2} d t,
$$

with $q_{i}$ and $q d_{i}$ the actual and desired positions of $i^{t h}$ joint. This cost function of figure 4 is here calculated off line first, for each subtask (among three) of the controller, according to the variation of the task periods (which may vary here from $0.5 \mathrm{~ms}$ to $30 \mathrm{~ms}$ ), the others remaining constant and fixed to $1 \mathrm{~ms}$. Notice that we have chosen to let the PD control task at a constant period, due to its high influence in the robot control stabilisation strategy. As done in [14], a variable period could have been assigned to this task to emphasise its importance in the closed-loop stability. However, in practical robot applications, it will remain at a constant rate. 


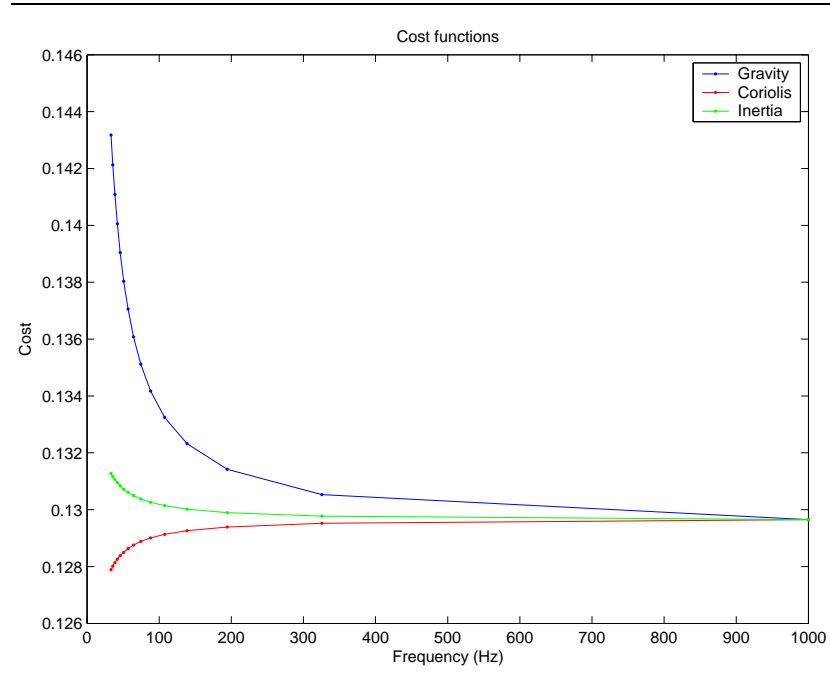

Figure 4. Cost functions

Based on this cost functions it appears that gravity compensation is the more important task therefore we have to allocate it more resources. Costs of Coriolis and inertia compensation are quite similar thus gravity compensation resources allocation is chosen to be twice of Coriolis or inertia ones.

In this application, the period of the feedback scheduler has been fixed to $30 \mathrm{~ms}$ to be larger than the robot control tasks (which limits have been fixed here from $0.5 \mathrm{~ms}$ to $30 \mathrm{~ms}$ ).

\subsection{Feedback scheduling design}

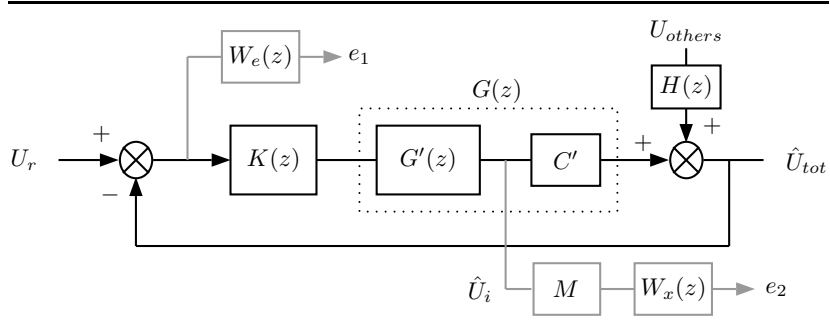

Figure 5. $H_{\infty}$ design bloc diagram

The bloc diagram of figure (5) is considered for the $H_{\infty}$ design where $G^{\prime}(z)$ is the model of the scheduler, the output of which is the vector of all task loads. To get the sum of all task loads, we have $C^{\prime}=\left[\begin{array}{lll}1 & 1 & 1\end{array}\right] . H(z)$ represents the sensor dynamical behaviour which measures the load of the other tasks. It may be a first or- der filter. The template $W_{e}$ specifies the performances on the load tracking error as follows :

$$
W_{e}(s)=\frac{s / M_{s}+\omega_{b}}{s+\omega_{s} \epsilon}
$$

with $M_{s}=2, \omega_{s}=10 \mathrm{rad} / \mathrm{s}, \epsilon=0.01$ to obtain a closed-loop settling time of $300 \mathrm{~ms}$, a static error less than $1 \%$ and a good robustness margin. Matrix $M=$ $\left[\begin{array}{lll}1 & -1 & -1\end{array}\right]$ and template $W_{x}$ allow to specify the load allocation between the control tasks. With a large gain in $W_{x}$, it leads to:

$$
U_{\text {gravity }} \approx U_{\text {Coriolis }}+U_{\text {inertia }},
$$

i.e. we allocate more resources for the gravity compensation.

All templates are discretized with a sampling period of $30 \mathrm{~ms}$. Finally discrete-time $H_{\infty}$ synthesis algorithm produces a discrete-time scheduling controller of order 4 .

\subsection{Simulations}

Simulations are performed using the TrueTime toolbox presented in [9]

Benchmark: The trajectory to be tracked consists in a point to point motion, coming from the position $[-\pi / 2,-\pi / 2,-\pi / 2,-\pi / 2,-\pi / 2,-\pi / 2,-\pi / 2]$ to $[\pi / 2,0, \pi / 2,0, \pi / 2,0, \pi / 2]$ in the joint space at a constant velocity for each joint. The trajectory duration is set to 2 secs, thus it is slow enough to avoid reaching the actuators limits. The system is observed on a total duration of 2.5 seconds.

Concerning feedback scheduling, the nominal set point of the resource utilisation is $60 \%$. At $t=1.5 \mathrm{~s}$, a disruptive task appears that needs $30 \%$ of the processor load.

Result analysis : From Fig. 6 (periods) the disruptive task produces a transient increase of the processor load at $t=1.5 \mathrm{~s}$. To reject this disturbance, the scheduling controller increases the periods of the three compensators. On Fig. 6 (loads) it appear that the gravity compensation load is twice those of the inertia or Coriolis load as specified through the $M$ and $W_{x}$ templates.

On Fig. 6 (Angle) we may check that the control load variations have no noticeable effects on the trajectory (as expected due to the co-design and feedback scheduling strategies), whereas the commands appear to be a little more noisy on Fig. 6 (torques).

Remark 1 As proposed in [14], the internal process controller can be also designed to take into account timing uncertainties, e.g. the control delays due to preemp- 

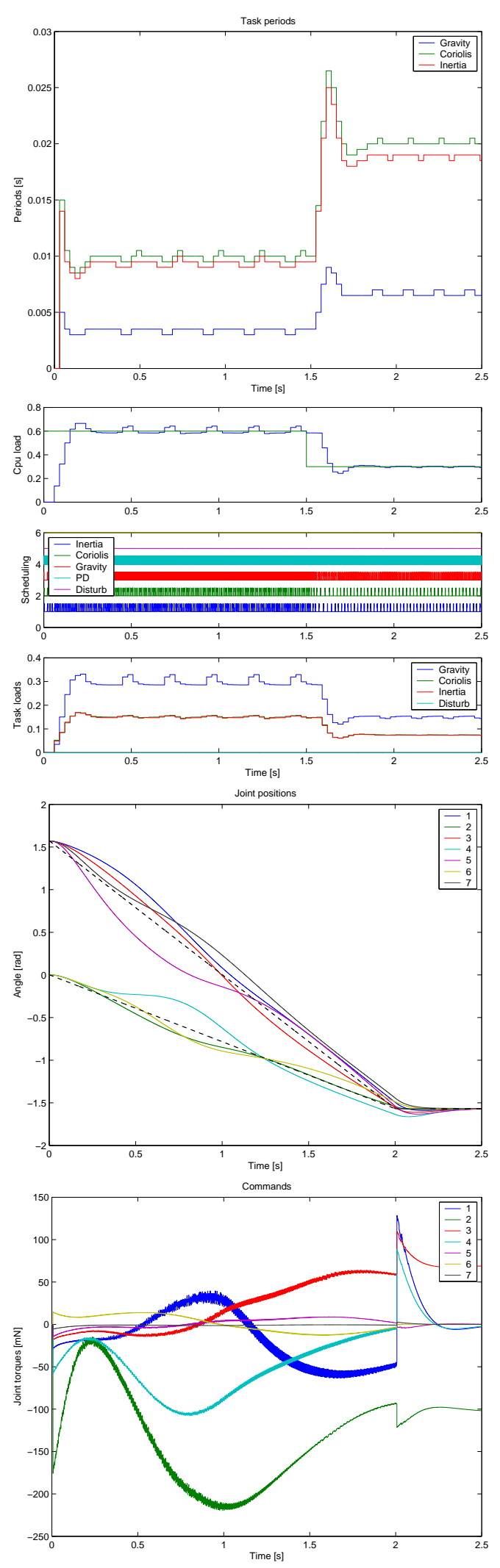

Figure 6. Simulations with TrueTime
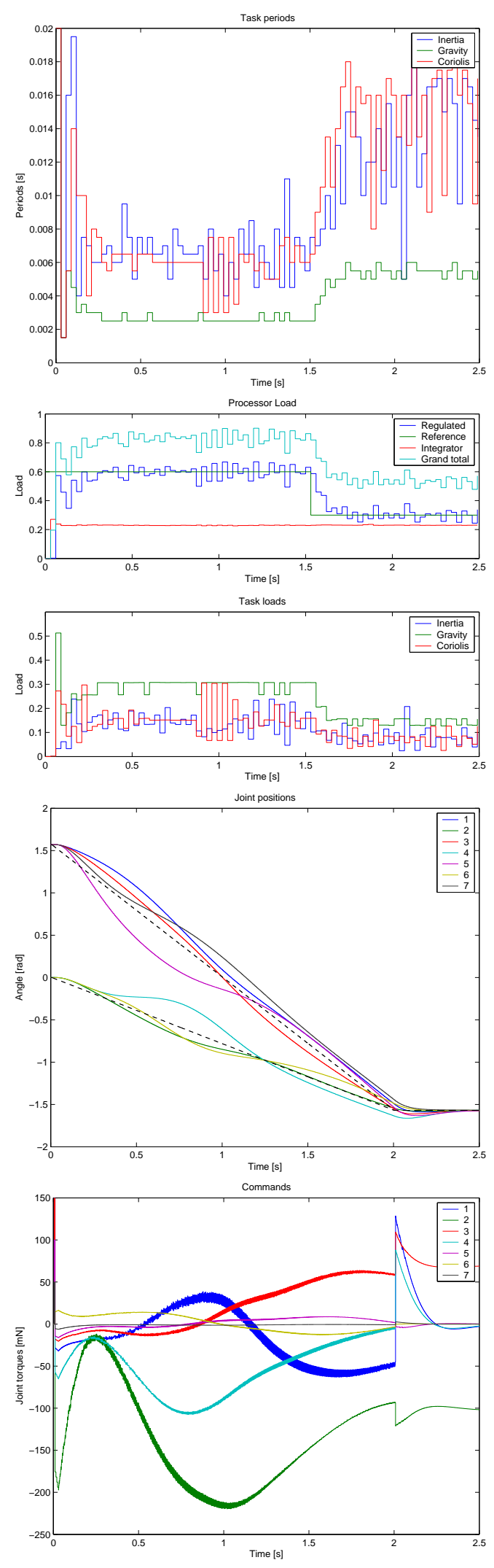

Figure 7. Experiments with RTAI 
tions which are unavoidable in real-time control and difficult to accurately predict in a dynamic environment.

\section{Feasibility of implementation for feedback schedulers}

While Truetime is very useful for fast prototyping and evaluation of scheduling controllers, it remains a simulation tool where the hardware and operating systems are abstracted. Execution times for control tasks are given as arbitrary values and execution times for the scheduler and system level housekeeping tasks are neglected. Therefore to assess the feasibility and practical interest of such adaptive closed-loop scheduling we have developed a feedback scheduler prototype on top of an existing robot controller. The current prototype runs under RTAI, the Realtime Linux Application Interface for Linux (http://www . aero polimi . it/ ${ }^{\sim}$ rtai/). This RTOS provides an efficient real-time scheduler (with a recorded interrupt latency range of $1--7 \mu s e c$ with the Pentium II $450 \mathrm{MHz}$ used for this setup) and allows time-stamping of events with the resolution of the built-in timer, thus the response times of each module can be accurately measured and plotted.

The original controller uses the so-called "Computing Torque Controller" which is split into several computing modules to implement a multi-rate controller as in [15] (Figure 8):

- CompTorque is the controller, updating the control torque vector $U$ from the state (position and velocity) error vector $Q_{d}-Q$ using a PD algorithm which gains are tuned to provide a comfortable stability margin. The sampling rate and latency of this block have a strong impact on the system's performance and stability. The extra inputs coming from the robot model's computations are used to provide feed-forward; in the previous simulation framework, it is called the PD control task. As well, the period of this task is constant for stability requirements.

- Gravity, Coriolis and Inertia compute an explicit model of the robot arm's dynamics. They are costly to compute but it has been shown through simulations that they can be executed several times slower than the main controller with a moderate impact on the control performance and stability;

- GeneTraj provides the desired trajectory viapoints at a fixed rate;

- The behaviour of the periodic control modules is supervised by a event driven reactive task in charge of setting up the system and exception handling, e.g. initialising the real-time tasks and cleanly stopping the system when it reaches the nominal termination state or when the control error exceeds a predefined threshold.

Control modules with different sampling rates communicate through asynchronous protected shared memories ; they are infinite loops triggered by clocks derived from the built-in timer by a middleware clock generator task (ClockGen).

As our goal is testing the feasibility of feedbackscheduling with no modifications of the internal of the operating system, the scheduling feedback loop is implemented as follows:

- The feedback scheduler is implemented as an additional real-time periodic task, i.e. a control module which function is specified and encoded by the control designer. The inputs are the measured response times of the control tasks. The set point is a desired global computing load. Outputs are the sampling periods of the Gravity, Coriolis and Inertia control tasks;

- The period of CompTorque is fixed in this particular experiment. It runs at a fixed period of $1 \mathrm{~ms}$ so that the stability of the system can be preserved. It implements the algorithm described in section 5.1 and reads the last available outputs of Gravity, Coriolis and Inertia via protected shared memories;

- The scheduling regulator adapts the periods of the Gravity, Coriolis and Inertia compensation modules and their computing load must be evaluated. The evaluation of the computing load is more or less easy and precise according the features of the operating system in use.

On one hand, the direct measure of tasks execution times is out of the scope of the API of a POSIX system. In that case, the computing load could be evaluated via the time of response of the tasks, but as the preemption phases cannot be discarded the global load would be over-estimated.

On the other hand, the RTAI kernel keeps track of the rt_tasks execution cycles, thus allowing to recover a precise measure of the control tasks execution times from the scheduling regulator.

Deadlines misses are checked and reported to the supervisor ; they are not taken into account in this preliminary setup but may be used in future improvements of a load estimator, for anti-windup and for overload handling;

- In this experiment the robot is still (cautiously) simulated: the drivers call a numerical integrator running the robot's dynamics model every time a 
measure is taken or a new control vector is sent by the CompTorque task. Therefore the controller and simulated process time scales are coherent, but the simulation adds unpredicted latencies in the loop. The corresponding measured added load is about $25 \%$ of the Pentium CPU used for the setup which is fortunately strongly over-sized for this robot controller;

- Priorities are ordered according to the relative urgency and weight of these function blocks on the system's behaviour: ClockGen $\succ$ Supervisor $\succ$ FedSched $\succ$ CompTorque $\succ$ GeneTraj $\succ$ Gravity $\succ$ Coriolis $\succ$ Inertia

- The initial periods has been set to $20 \mathrm{msec}$ for the three tasks with a variable and controlled period, i.e. Gravity, Coriolis and Inertia. The PD control task is run at a fixed rate of $1 \mathrm{~ms}$ and the trajectory generator provides new set points every $5 \mathrm{~ms}$. The feedback scheduler is run every $30 \mathrm{msec}$.

- The sampling frequency of the clock generator is set to $2 \mathrm{KHz}$ thus allowing to increment or decrement the control tasks clocks by $500 \mu \mathrm{sec}$ steps. Observing and managing the system at a faster rate would induce a very high system's load due to too many context switches.

- The desired trajectory is the same as in 5. Preliminary experiments shown that the robot's numerical integration spends about $25 \%$ of the available CPU power. As we need some load margin to avoid transient overruns the desired load is initially set to 0.6 . At time $1.5 \mathrm{sec}$ it is decreased to 0.3 to make room for a disturbing incoming task proposed for admission.

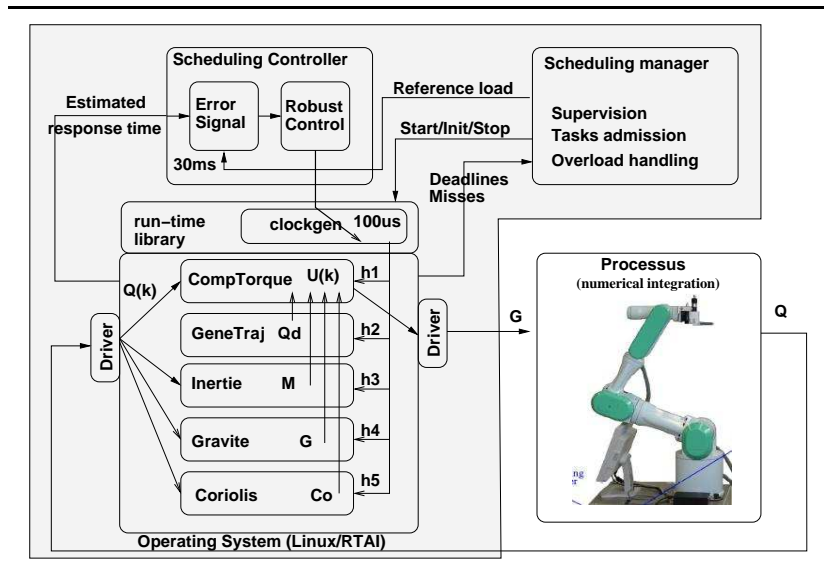

Figure 8. Feed-back scheduling experiment

As plot in Figure 7 the very first experimental results are encouraging: they show that such a feedback scheduling architecture can be quite easily designed and implemented on top of an off-the-shelf real-time operating system with fixed priority and preemption. Examining the plots and the kernel reporting file calls for the following comments:

- As the feedback scheduler is a simple feedback algorithm running at a slow rate its computing cost is quite low (about $75 \mu \mathrm{sec}$ every $30 \mathrm{~ms}$ ). On the other hand observing and managing the system at a high rate can be very costly due to numerous context switches. A more efficient implementation is currently studied to decrease the system's cost.

- The tasks loads can be directly measured using a specific, non portable feature of the RTAI kernel. As response times are easier to measure (e.g.from a POSIX API), using an execution time estimator would increase the portability of the system. Designing such a low-cost and reliable estimator remains to be done.

- Overshoots in the control periods and load lead to transient overload and deadline misses. These events are not currently processed leading to an avalanche of timing faults and to a system unstability and failure. Overruns and other timing errors must be adequately processed at the supervision level to more safely manage the system and make use of the full range of available processing power.

\section{Conclusion}

In this paper, a methodology for control and scheduling co-design is proposed. Besides the usual modelling errors in the process model a digital closed-loop controller is also submitted to timing disturbances coming from the implementation: they are difficult to be accurately predicted and studying the impact of timing deviations in feedback loops is still a largely unexplored domain. Hence a natural idea consists in using robust control theory to design controllers to be weakly timing sensitive. Besides process control this idea can be used also to design a feedback scheduling loop to implement robust on-line adaption of the scheduling parameters according to estimates of the computing activity. Thus this resource allocation control loop is used to fulfil the plant control objective under constraint of limited computing resource.

An integrated control-scheduling framework is proposed. The control periods are weighted according to their impact on the control performance. An outer scheduling controller then regulates in real-time the 
CPU load according to the allocated computing power. Indeed the control synthesis of the feedback scheduler has been provided using the $H_{\infty}$ control theory, and the gain of the controller is adapted on-line using the estimated execution times of the control tasks. In all cases the effectiveness of the controllers lies in a right choice of the design weighting functions used to specify the trade-off between concurrent constraints. Therefore the designer(s) must have knowledge on both the process and computing platform capabilities to be able to best fit the end-user requirements.

Some simulation and experiment results have been given, which emphasises the interest of this approach and a software prototype has been designed to assess the feasibility of the approach using an off-the-shelf real-time operating system.

Further works concern the improvement of robust control schemes for both the process and scheduler controllers. A better insight in control w.r.t. timing uncertainties will be necessary to efficiently shape the weighting between control and computing constraints. Implementation feasibility must be taken into account and the system's supervision must be improved. Finally choosing strategies and tuning parameters leading to an effective trade-off which fit with the end-user's requirements needs a common understanding and cooperation between control and computer scientists and engineers.

\section{References}

[1] K. Åström and B. Wittenmark. Computer-Controlled Systems. Information and systems sciences series. Prentice Hall, New Jersey, 3rd edition, 1997.

[2] G. Bernat, A. Burns, and A. Llamosí. Weakly hard real-time systems. IEEE Transactions on Computers, 50(4):308-321, 2001.

[3] G. Buttazzo and L. Abeni. Adaptive rate control through elastic scheduling. In 39th Conference on Decision and Control, Sydney, Australia, 2000.

[4] A. Cervin. Integrated Control and Real-Time Scheduling. PhD thesis, Department of Automatic Control, Lund Institute of Technology, Sweden, Apr. 2003.

[5] A. Cervin and J. Eker. Feedback scheduling of contol tasks. In Proceedings of the 39th IEEE Conference on Decision and Control, Sydney, Australia, Dec. 2000.

[6] A. Cervin, J. Eker, B. Bernhardsson, and K.-E. Årzén. Feedback-feedforward scheduling of control tasks. RealTime Systems, 23(1), 2002.
[7] J. Eker, P. Hagander, and K.-E. Årzén. A feedback scheduler for real-time control tasks. Control Engineering Practice, 8(12):1369-1378, 2000.

[8] J. Eker, P. Hagander, and K.-E. Årzén. A feedback scheduler for real-time controller tasks. Control Engineering Practice, 8(12):pp 1369-1378, 2000.

[9] D. Henriksson, A. Cervin, and K.-E. Årzén. Truetime: Simulation of control loops under shared computer resources. In 15th IFAC World Congress on Automatic Control, Barcelona, Spain, July 2002.

[10] C. Lu, J.-A. Stankovic, G. Tao, and S.-H. Son. Feedback control real-time scheduling: Framework, modeling, and algorithms. Real Time Systems, 23(1):85-126, 2002.

[11] M. Ryu, S. Hong, and M. Saksena. Streamlining realtime controller design: from performance specifications to end-to-end timing constraints. In IEEE Real Time Systems Symposium, 1997.

[12] K. Sandström and C. Norström. Managing complex temporal requirements in real-time control systems. In 9th IEEE Int. Conf. and Workshop on the Engineering of Computer-Based Systems (ECBS'02), Lund, Sweden, 2002.

[13] M. Schinkel, W.-H. Chen, and A. Rantzer. Optimal control for systems with varying sampling rate. In Proceedings of American Control Conference, Anchorage, May 2002.

[14] O. Sename, D. Simon, and D. Robert. Feedback scheduling for real-time control of systems with communication delays. In ETFA'03 9th IEEE International Conference on Emerging Technologies and Factory Automation, Lisbonne, september 2003.

[15] D. Simon, E. Castillo, and P. Freedman. Design and analysis of synchronization for real-time closed-loop control in robotics. IEEE Trans. on Control Systems Technology, 6(4):445-461, july 1998.

[16] D. Simon, K. Kapellos, and B. Espiau. Control laws, tasks and procedures with orccad: Application to the control of an underwater arm. Int. Journal of Systems Science, 29(10):1081-1098, 1998.

[17] D. Simon, O. Sename, D. Robert, and O. Testa. Realtime and delay-dependent control co-design through feedback scheduling. In CERTS'03 Workshop on Codesign in Embedded Real-time Systems, Porto, july 2003. ECRTS.

[18] M. Törngren. Fundamentals of implementing real-time control applications in distributed computer systems. Real Time Systems, 14(3):219-250, 1998.

[19] K. Zhou, J. C. Doyle, and K. Glover. Robust and optimal control. Prentice-Hall Inc., 1996. 